\title{
ALteraÇÕES DA MOTILIDADE ANTRODUODENAL E DO PH GÁSTRICO APÓS RESSECÇÃO GáSTRICA PARCIAL INCLUINDO A ÁREA CORRESPONDENTE AO MARCA-PASSO DO ESTÔMAGO DE CÃES
}

\author{
Paulo Afonso Nunes Nassif, Karl-Hermann Fuchs, Johannes Heimbucher, Osvaldo Malafaia, \\ Nicolau Gregori Czeczko, Jurandir Marcondes Ribas filho, Arnulf Thiede, Ulrich Andreas Dietz* \\ Trabalho realizado no Programa de Pós-graduação em Clínica Cirúrgica da Universidade Federal do Paraná em cooperação com o Instituto de \\ Pesquisas Médicas da Faculdade Evangélica de Medicina do Paraná e a Clínica Cirúrgica da Universidade de Würzburg - Alemanha.
}

RESUMO - As alterações funcionais da motilidade antroduodenal ainda não foram bem caracterizadas até a presente data. 0 marca-passo gástrico, possivelmente localiza-se junto à grande curvatura, próximo ao fundo gástrico. A partir dele, ondas de despolarização dirigem-se ao estômago, duodeno e às demais partes do intestino delgado.

ОвеEтIvo. Avaliar a influência do marca-passo gástrico sobre a motilidade gastroduodenal em cães.

Métodos. 0 protocolo experimental constou de três etapas. Na primeira, realizou-se esofagostomia cervical seguida de manometria antroduodenal eletrônica e pH-metria em 20 cães (grupo controle), durante 4 horas. Na segunda etapa, realizou-se a ressecção de parte do fundo gástrico e da área do marca-passo gástrico, com técnica videolaparoscópica. $\mathrm{Na}$ terceira, decorridos três meses, repetiu-se a manometria antroduodenal e a pH-metria gástrica.

Resultados. No grupo controle observou-se traçado típico de motilidade antroduodenal, com complexo interdigestivo de motilidade (IMC) com fases I, 2 e 3; na pH-metria, observouse padrão de acidez com linha de base em pH de I, 15. Após a ressecção gástrica parcial, a manometria apresentou, no geral, aumento na freqüência de contrações, aumento da participação relativa da fase 2 e diminuição da participação relativa da fase 3. No pH gástrico, observou-se aumento no percentual de $\mathrm{pH} 0$ - I e pH I - 2, bem como diminuição na porcentagem de $\mathrm{pH} 6-7$, com conseqüente diminuição do $\mathrm{pH}$ gástrico e redução no tempo prandial.

Conclusão. Pela retirada da área do marca-passo gástrico ocorreram alterações de motilidade antroduodenal, permanecendo porém a ocorrência de IMC's completos.

UnITERMOS: Marca-passo gástrico. Manometria antroduodenal. $\mathrm{pH}$-metria. Videolaparoscopia. Cães.

\section{INTRODUÇÃO}

Nos últimos anos, alterações de motilidade do sistema digestório passaram a ocupar posição de destaque na pesquisa em gastroenterologia clínica e cirúrgica ${ }^{1-6}$. Através de equipamentos de eletromiografia e manometria é possível mapear padrões fisiológicos de motilidade do trato gastrointestinal. Alterações funcionais da motilidade antroduodenal, todavia, não foram bem caracterizadas e definidas até a presente data. Na prática clínica, as alterações funcionais antroduodenais evoluem com sintomas inespecíficos; é comum que sejam chamados de dispepsia não-ulcerosa ou dispepsia crônica, quadros clínicos estes sem definição exata e sem critérios diagnósticos precisos ${ }^{7,8}$. As principais

\footnotetext{
*Correspondência:

Chirurgische Universitätsklinik und Poliklinik Würzburg Josef-Schneider-Strasse, 2 97080 Würzburg - Alemanha

Fone: (49) 9312011 - Fax.: (49) 9312013225
}

dificuldades no estudo destes quadros são por um lado a dificuldade do diagnóstico fisiopatológico das alterações de motilidade antroduodenal, e por outro lado a inespecificidade dos sintomas relacionados.

O funcionamento rítmico e coordenado das regiões gástricas é coordenado pelo marca-passo gástrico ${ }^{4}$. O marca-passo gástrico localiza-se, possivelmente, junto à curvatura gástrica maior, próximo ao fundo gástrico e é autônomo na geração de despolarizações cíclicas, numa freqüência de até três contrações por minuto. A partir dele, as ondas de despolarização passam por todo o estômagoe chegam até o duodeno, e as demais partes do intestino delgado-12.

Após o esvaziamento gástrico, inicia-se uma etapa de atividade motora - apesar de o estômago estar praticamente vazio-conhecida como complexo motor interdigestivo. Esta atividade motora ocorre tanto no estômago quanto no duodeno $0^{13,14}$. Durante esta etapa, contrações gástricas vigorosas, associadas a aberturas amplas do piloro, realizam a limpeza do estômago, de restos alimentares que não tenham sido bem fragmentados na fase pósprandial| $^{13,15,16}$. Este padrão se repete em praticamente todos os mamíferos. A etapa interdigestiva do cão divide-se em três fases distintas de motilidade gástrica: a fase I é caracterizada por ausência de atividade motora e dura de 45 minutos a 60 minutos; a fase 2 apresenta contrações intermitentes, com freqüência de $\mathrm{I}-2 /$ minuto em antro gástrico e de 2-5/minuto em duodeno, e dura aproximadamente 30 minutos; e a fase 3 desenvolve contrações rítmicas que elevam a pressão intragástrica ao seu maior patamar, com freqüência de 2,5-3,5/minuto no antro e de 8-12/minuto no duodeno, dura de 5 minutos a 10 minutos; a fase 3 é conhecida por complexo motor migratório (migrating motor complex, MMC). As três fases seguem uma a outra, compondo um ciclo com 70 minutos a 140 minutos de duração; este ciclo completo das fases I, 2 e 3 é conhecido por complexo 
motor interdigestivo (interdigestive motor complex, IMC) ${ }^{13}$. As três fases do IMC iniciamse no estômago e se propagam até o íleo terminal. Ocorrendo ingestão alimentar, 0 IMC éabruptamente interrompido, iniciandose o padrão prandial de motilidade ${ }^{15,17}$.

\section{Métodos}

Para permitir o estudo manométrico antroduodenal sem a necessidade de se ficar em posição de decúbito ao lado de complicados equipamentos, foi desenvolvido sistema com sonda eletrônica dotada de diversos pontos de medição e capaz de registrar os dados durante 24 horas em aparelho portátil anexo. Utilizando este sistema eletrônico, o presente estudo objetiva registrar o padrão de motilidade antroduodenal e pH-metria gástrica em cães não operados do estômago; e estudar as alterações da motilidade antroduodenal e de pH-metria gástrica após a ressecção da área correspondente ao marca-passo gástrico e parte do reservatório do estômago.

Utilizou-se 20 cães mestiços e o protocolo experimental constou de três etapas: primeira, realização de esofagostomia cervical seguida de manometria antroduodenal e $\mathrm{pH}$-metria gástrica após 15 dias; segunda, ressecção da parte do fundo gástrico correspondente a parte do reservatório e área do marca-passo gástricos; terceira, decorridos três meses da ressecção, realizou-se novo estudo de manometria antroduodenal e $\mathrm{pH}$-metria gástrica.

O sistema para manometria antroduodenal constou de sonda e aparelho de registro contínuo de dados. A sonda de manometria antroduodenal possui seis pontos eletrônicos para aferição de pressão e tem diâmetro de $4,5 \mathrm{~mm}$ no local dos sensores e de $2,0 \mathrm{~mm}$ nos demais segmentos. Os quatro sensores proximais foram definidos para 0 estudo da manometria em antro gástrico e distam, entre $\mathrm{si}, 2 \mathrm{~cm}$. Os dois sensores definidos para a manometria duodenal localizam-se a $5 \mathrm{~cm}$ e $15 \mathrm{~cm}$ de distância do sensor mais distal do antro gástrico (Konigsberg Instruments). A calibração foi realizada com os sensores expostos à pressão atmosférica (zero) e sob pressão pneumática de $50 \mathrm{mmHg}$. Os dados foram armazenados em equipamento "Microdigitrapper" com 4MB (Synectics) e analisados no programa "Multigram" versão $6.3 \mathrm{IH}$ (Gastrosoft).
$\mathrm{OpH}$ foi determinado mediante eletrodo de antimônio com diâmetro de $2 \mathrm{~mm}$ e os dados da $\mathrm{pH}$-metria foram registrados por equipamento portátil de $\mathrm{pH}$-metria, modelo "Digitrapper Mark II Gold" (Synectics). A análise dos dados foi feita pelo programa "esophoGram" versão 5.7 (Gastrosoft). Calibrou-se o sistema de $\mathrm{pH}$-metria mediante duas soluções padrão com pH preestabelecido em I e 7. Para a análise dos resultados definiu-se linha de base como sendo a linha relativa ao valor mais freqüente de $\mathrm{pH}$ abaixo de 2; identificou-se os períodos prandial (platô) e pós-prandial (declive) segundo o Gráfico I, considerando-se o aumento do $\mathrm{pH}$ logo após a ingestão alimentar como início do período prandial; com a queda do $\mathrm{pH}$ iniciavase o período pós-prandial. Agrupou-se os dados em porcentagem de tempo no qual o $\mathrm{pH}$ ficava entre os valores de 0 - I, I-2, 2-3, 3-4, 45, 5-6, 6-7 e pH maior do que $7^{2}$.

Para os procedimentos cirúrgicos deste experimento utilizou-se anestesia geral com ventilação assistida. O pré-anestésico constou de clorpromazina $(0,5 \mathrm{mg} / \mathrm{Kg} \mathrm{SC})$ e sulfato de atropina $(0,01 \mathrm{mg} / \mathrm{Kg})$. Aanestesia foi induzida com solução de pentobarbital sódico $(5 \mathrm{mg} /$ $\mathrm{Kg})$ e citrato de fentanil $(2,5 \mu \mathrm{g} / \mathrm{Kg})$, até o relaxamento da mandíbula. A seguir, o cão era posicionado na mesa cirúrgica, intubado e mantido em respiração assistida. A manutenção anestésica foi feita com vaporização de Halotano ${ }^{\circledR}$ a $2 \%$. Realizou-se prevenção antibiótica com $500 \mathrm{mg}$ de cefoxitina sódica EV.

Como etapa preparatória (primeiro procedimento cirúrgico), realizou-se esofagostomia cervical em todos os animais, para que se pudesse posicionar as sondas de manometria antroduodenal e $\mathrm{pH}$-metria previstas no protocolo. $O$ acesso foi cervicotomia longitudinal de $4 \mathrm{~cm}$ junto à borda ventral do músculo esternocleidomastóideo esquerdo. Descolou-se o esôfago cervical de seu leito, incisou-se a borda lateral esquerda do esôfago numa extensão de $2 \mathrm{~cm}$ efixou-se as bordas da abertura esofágica à pele com 8 pontos simples, separados. Após 24 horas os animais passaram a receber água e ração comercial balanceada para a espécie ad libitum.

Após 15 dias avaliou-se a motilidade antroduodenal e a $\mathrm{pH}$-metria gástrica para obtenção de valores de referência com estômago não operado (grupo controle). Para posicionamento das sondas de manometria e
pH-metria, o animal era submetido a sedação endovenosa com tiopental sódico. Com auxílio de gastroscópio flexível com visão lateral (Olympus KF I0), posicionou-se a sonda de manometria antroduodenal com dois canais de medição no duodeno e quatro no antro gástrico. A sonda de $\mathrm{pH}$-metria gástrica foi posicionada com o eletrodo a $5 \mathrm{~cm}$ distal ao pinçamento diafragmático do esôfago; afixouse eletrodo de referência à pele. A seguir, 0 animal era colocado numa armação de alumínio dotada de rodinhas (ambulante), a qual permitia que durante as fases de medição e coleta de dados o animal se locomovesse livremente pelo laboratório, sem danificar ou deslocar as sondas de medição. Os aparelhos de registro ("Microdigitrapper" e "Digitrapper") eram afixados à estrutura do ambulante. Iniciava-se o registro dos dados 30 minutos após a sedação, tempo no qual todos os animais haviam recuperado as condições habituais de atividade física. $O$ tempo total de registro para a manometria antroduodenal e para a $\mathrm{pH}$-metria gástrica foi de 4 horas: durante as primeiras três horas de medição o animal permanecia em jejum; a seguir, recebia $100 \mathrm{~g}$ de ração e $200 \mathrm{ml}$ de água e passava-se a registrar os dados por mais uma hora.

Realizou-se a ressecção gástrica parcial mediante técnica de videocirurgia (segundo procedimento cirúrgico). Instalou-se pneumoperitônio("Electronic Laproflator 264300 2C" STORZ) e posicionou-se quatro trocartes: um de $10 \mathrm{~mm}$ para a óptica de $30^{\circ}$ (quadrante caudal direito do abdome) e os demais de 15, 5 e $10 \mathrm{~mm}$, respectivamente, em quadrante caudal esquerdo do abdome (para ogrampeador endoscópico), quadrante cranial direito do abdome e quadrante cranial esquerdo do abdome. Liberou-se o fundo gástrico e realizou-se ressecção gástrica envolvendo parte da curvatura gástrica maior e do fundo do estômago; este segmento compreenderia a topografia do marca-passo gástrico. Os limites desta ressecção foram do terço cranial da curvatura gástrica maior até I $\mathrm{cm}$ de distância da transição esofagogástrica. Utilizouse grampeador linear cortante endoscópico ("Endo-Gia 60", Auto Suture), com três a quatro disparos.

Três meses após a realização da ressecção gástrica parcial, reposicionou-se as sondas para realização da manometria antroduodenal e da pH-metria gástrica pós-operatórias. 


\section{Gráfico I - Definição das fases prandial e pós-prandial pela pH-metria}

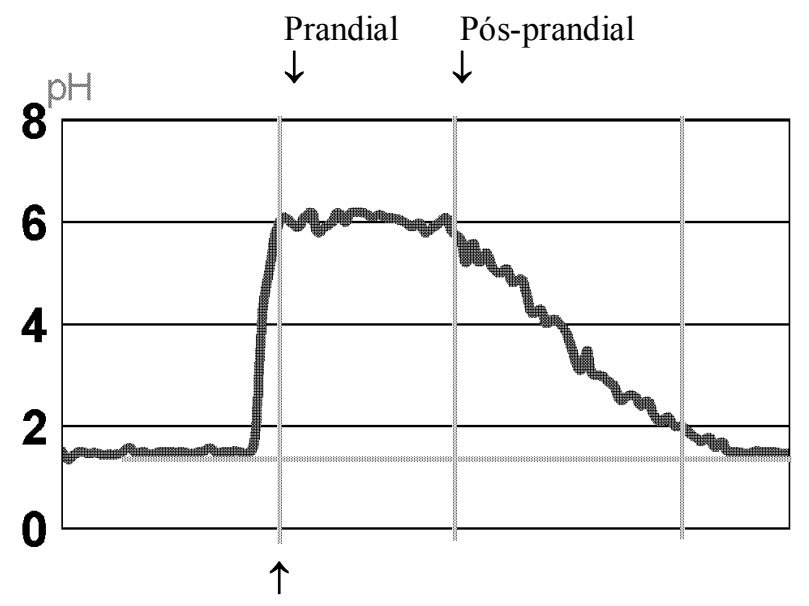

Linha de base do $\mathrm{pH}$

Alimentação
Gráfico 2 - Amplitude e participação das fases I a 3 em antro gástrico (em \%), antes (pré-operatório) e após (pós-operatório) a ressecção gástrica parcial incluindo a área do marca-passo do estômago em cães. Fases $I+2+3=I M C$. No pós-operatório, o tempo total do IMC está aumentando, a particpação da fase 2 é significativamente maior $(p=0,002)$ e a amplitude da fase 3 é maior $(p=0,0125)$

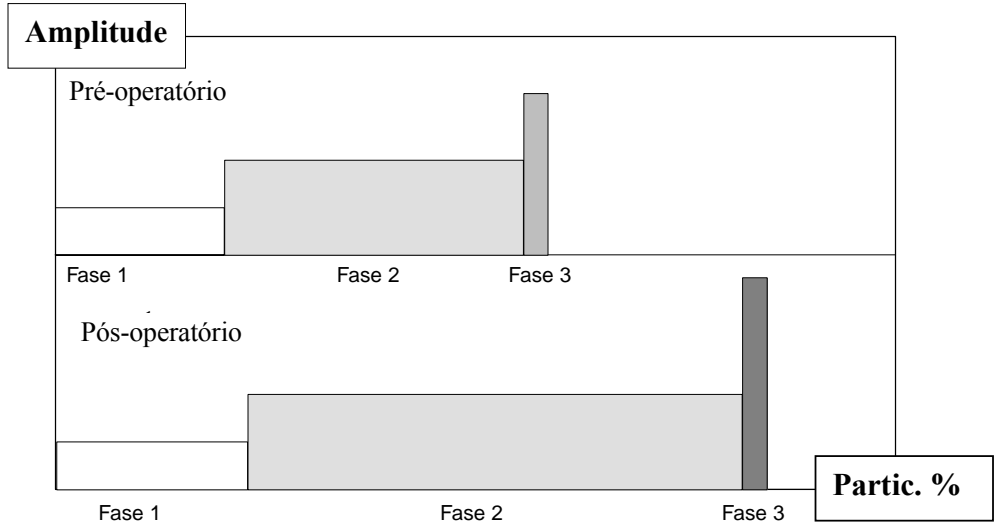

O tratamento estatístico dos dados constou do cálculo dos valores medianos e os percentis de $5 \%$ e $95 \%$ dos resultados e, dependendo da natureza dos dados, pelo teste " $\mathrm{t}$ " (amostras pareadas), ou pelo teste de WILCOXON (amostras não pareadas), utilizando-se o programa "InStat"(GraphPad). Considerou-se como valores estatisticamente significativos aqueles que tiveram 0 valor de "p" calculado como menor do que $5 \%$ ou 0,05 . Nos animais não operados do estômago (grupo controle), observou-se nas fases interdigestivas do antro gástrico em média dois IMC's, com tempo médio de 69 minutos cada um; a freqüência de contrações durante a fase 3 foi de 3,05 contrações por minuto; na média geral das fases I a 3, a freqüência foi de $I, 07$ contração por minuto; no período pósprandial, a freqüência de contrações foi I,87 por minuto. No duodeno, observaram-se em média dois IMC's durante a fase interdigestiva; a frequiência de contrações durante a fase 3 foi de $11,76 / \mathrm{min}$, com freqüência média de contração na média geral nas fases de 4,04 / min; a freqüência de contração durante o período pós-prandial foi de 3,82 / min.

Após a ressecção do marca-passo gástrico, foram observados alterações significativas em I 5 dos 49 parâmetros estudados, sendo nove na manometria do antro gástrico e seis na manometria do duodeno. No antro gástrico observou-se a ocorrência das seguintes alterações: ocorreu aumento estatisticamente significativo na freqüência de contrações na média geral nas fases ( $I, 07$ contração por minuto no pré e 1,26 contração por minuto no pósoperatório, $p=0,01$ ); a participação da fase 2 aumentou de $51,5 \%$ para $57,5 \%$, respectivamente $(p=0,002)$; a participação das fases I ( $24,5 \%$ na pré-operatório e $18 \%$ no pósoperatório, $p=0,006)$ e 3 (3,63\% no préoperatório contra $2,55 \%$ no pós-operatório, $\mathrm{p}=0,002$ ) diminuíram significativamente, respectivamente; além disso, a fase 3 apresentou significativa redução no tempo de duração ( 3,05 segundos no pré-operatório e 2,85 segundos no pós-operatório, $p=0,0 \mathrm{I}$ ), aumento na freqüência de contrações $(3,35$ no préoperatório contra 4,17 no pós-operatório, $p$ $=0,00 \mathrm{I}$ ), assim como aumento significativo na amplitude de contração (47, I 3 no pré-operatório contra $5 \mathrm{I}, 48 \mathrm{mmHg}$ no pós-operatório, $p=0,0125)$; as alterações observadas nas fases apresentaram tendência de prolongamento da IMC (69 pré-operatório contra 103 minutos pós-operatório), porém com significância estatística limítrofe $(p=0,05)$; observou-se ainda aumento significativo na amplitude de contração durante a fase pós-prandial (24,42 pré-operatório contra $31,50 \mathrm{mmHg}$ pós-operatório, $p=0,0 I$ ). 0 Gráfico 2 demonstra as alterações de amplitude e participação das fases I, 2 e 3 no antro gástrico, antes e após a ressecção gástrica parcial.

No duodeno observou-se alterações significativas em seis parâmetros: os IMC's apresentaram diminuição no tempo de duração (62 pré-operatório para 45 minutos pósoperatório, $p=0,002$ ); na fase 2, observou-se um aumento significativo na freqüência de contrações (4,56 pré-operatório para $5,32 \mathrm{n}$ / min pós-operatório, $p=0,02$ ); a participação relativa da fase 3 nos IMC's apresentou-se significativamente diminuída (I 2,5 pré-opera- 
Gráfico 3 - Amplitude e participação das fases I a 3 em duodeno (em \%), antes (pré-operatório) e após (pós-operatório) a ressecção gástrica parcial incluindo a área do marca-passo do estômago em cães. Fases $I+2+3=I M C$. No pós-operatório, o tempo total do IMC está significativamente reduzido $(p=0,002)$, a participação relativa da fase está aumentada $(p=0,002)$ e a participação relativa dafase 3 , diminuída $(p=0,0027)$

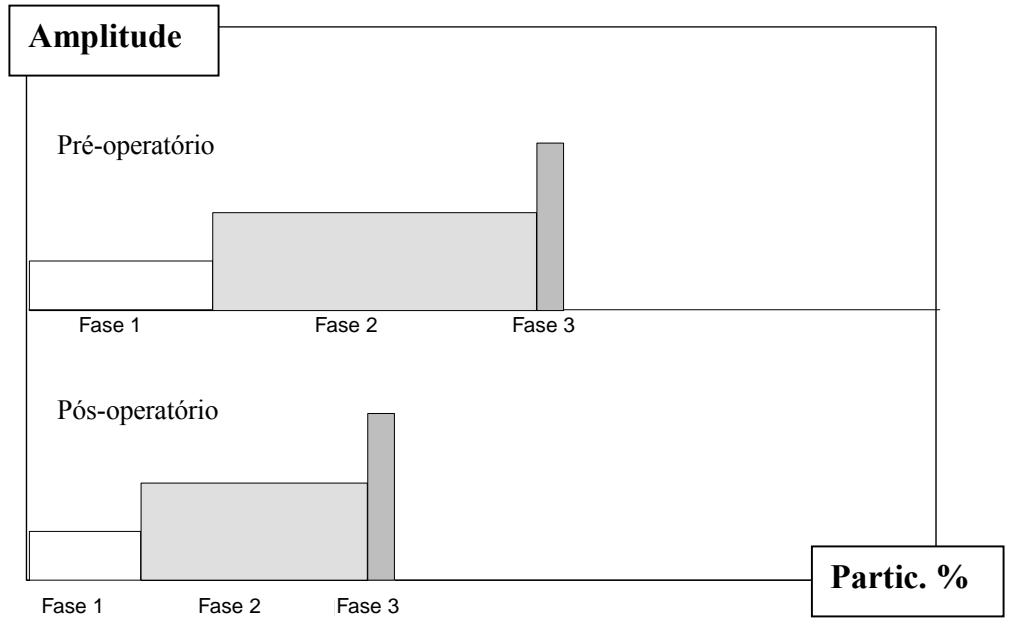

Gráfico 4 - Resultados comparativos da pH-metria no grupo controle e no pós-operatório

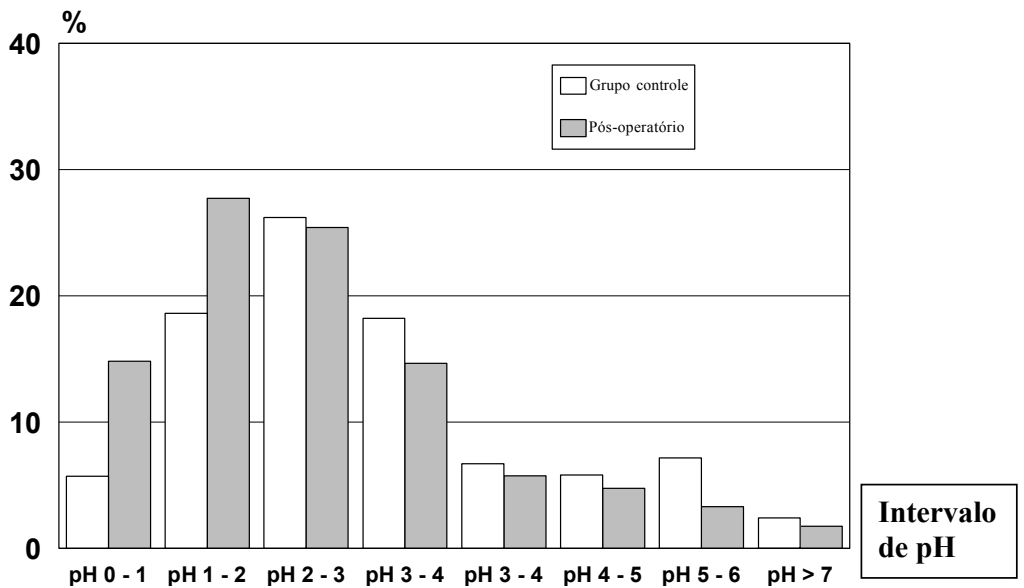

tório para $11,52 \%$ pós-operatório, $\mathrm{p}=$ $0,003)$; na média geral nas fases houve aumento da freqüência de contrações (4,04 préoperatório para 4,55 pós-operatório contrações por minuto, $p=0,0201$ ), aumento da participação relativa da fase $2(62,35 \%$ préoperatório para $71,53 \%$ pós-operatório, $p=$ 0,0021 ) e diminuição da participação relativa da fase 3 (3,33 pré-operatório para 2, I4\% rio e $\mathrm{pH} \mathrm{I,00} \mathrm{no} \mathrm{pós-operatório,} \mathrm{p}=0,07$ ). $\mathrm{O}$ tempo percentual de $\mathrm{pH}$ foi significativamente maior nos intervalos de medição de percentagem de $\mathrm{pH} 0$ - I e percentagem de $\mathrm{pH} \mathrm{I}$ - 2, no pós-operatório (5,7\% pré-operatório para $14,8 \%$ pós-operatório com $\mathrm{p}=$ 0,01; e 18,6\% no pré-operatório e $27,65 \%$ no pós-operatório, com $p<0,001$, respectivamente; houve diminuição significativa no tempo percentual de $\mathrm{pH} 6-7$, de 7, I5\% préoperatório para $3,3 \%$ pós-operatório $(p=$ $0,003)$. Observou-se redução significativa do tempo prandial de 12,5 no pré-operatório para 7,5 minutos no pós-operatório $(p<$ 0,002) (Gráfico 4).

\section{Discussão}

A motilidade do estômago e do duodeno apresenta padrão constante, semelhante em praticamente todos os mamíferos, sendo qualitativamente comparável entre o homem e o cão $13,16,17,18,19$. O segmento antroduodenal tem um padrão de motilidade típico. O período de descanso gástrico é conhecido como fase interdigestiva. Quando o estômago está vazio (período interdigestivo), observa-se o IMC; durante o IMC, alterações mínimas de motilidade sensibilizam os sensores da manometria intraluminar, permitindo a diferenciação típica das fases I, 2 e 3. Quando o estômago está cheio, não é possível a medição manométrica de pequenas alterações de pressão devido à interferência do conteúdo alimentar (padrão prandial e pós-prandial, definido pela ausência do padrão de motilidade interdigestivo).

Provavelmente, há influência dos troncos vagais sobre a motilidade gástrica, porém não são eles os determinantes da mesma. Gleysteen, Sarna e Myrvik ${ }^{20}$ relataram permanência de $90 \%$ dos IMC's após resfriamento dos troncos vagais, sendo o efeito modulador dos mesmos sobre a motilidade gástrica notada no aumentando do tempo da fase 3; ressaltam que descargas vagais não têm a capacidade de iniciar um IMC, nem alterar sua duração total. Resultados semelhantes foram relatados por Sarna, Stoddard, Belbeck et al ${ }^{21}$, demonstrando que iniciação e migração dos IMC's em intestino delgado de cães estão na dependência da rede neuronal intramural e não de comandos vagais extrínsecos ${ }^{15,20,22,23}$.

Alterações funcionais de esvaziamento 
NASSIF PAN ET AL.

gástrico podem ser causadas por diminuição do tônus do fundo gástrico, por atividade alterada do antro gástrico ou ainda por alterações de coordenação entre antro e duodeno ${ }^{3}$. Os quadros melhor estudados são aqueles relacionados a neuropatias, como diabetes melito e esclerose múltipla ${ }^{24}$, os relacionadas à desnervação do trauma raquimedular ${ }^{25}$, ou ainda os relacionadas a estados pós-vagotomia ${ }^{26,27}$. Alterações da motilidade interdigestiva também podem causar desconforto, sem estar relacionadas a doenças conhecidas $^{7,26}$. O presente estudo experimental busca aproximar-se dessa lacuna do conhecimento.

Agrande maioria dos estudos experimentais sobre motilidade antroduodenal disponíveis na literatura têm o cão como modelo animal| ${ }^{13,18,28-31}$. Segundo levantamento da bibliografia atéa data do presente experimento, o procedimento cirúrgico empregado nesse estudo é original quanto à sua proposição fisiológica. A realização da ressecção gástrica parcial teve como finalidade a retirada da área correspondente ao marca-passo gástrico na porção cranial da curvatura gástrica maior, e para facilitar sua padronização, envolveu a ressecção da cúpula do fundo gástrico. Assim, esperam-se dois efeitos pós-operatórios: primeiro, a redução do volume do fundo gástrico e redução da capacidade de recepção de alimentos; e segundo, a supressão da área dominante do marca-passo gástrico.

A retirada da área geradora de impulsos dominante na despolarização gástrica pode ser responsável por alterações no IMC. Com o modelo cirúrgico aqui proposto, manteve-sea integridade do piloro como barreira natural contra o refluxo duodenogástrico, restando analisar se o estômago residual é capaz de desenvolver motilidade fisiologicamente eficiente para assumir as atividades digestivas e interdigestivas.

A existência e a localização topográfica da área geradora dos impulsos de marca-passo gástrico foi intensamente estudada por Weber e Kohatsu': mapeando o estômago de cães, os autores identificaram a região anatômica do marca-passo gástrico na porção cranial da curvatura maior do estômago como parte das fibras musculares longitudinais e demonstraram que a parte proximal do corpo gástrico é dominante quanto à atividade elétrica.

Durante várias décadas o registro da motilidade antroduodenal foi realizado experimentalmente pela técnica da eletromiografia, $93,15,16,17,19,20,22 \mathrm{e}$, mais recentemente (também com aplicação clínica), pela manometria de perfusão $0^{3,25,32,33}$. Essaúltima revolucionou a possibilidade de registros de motilidade, sem a necessidade de acessos cirúrgicos, porém tem a desvantagem de não permitir medições contínuas e por tempos prolongados, pois isso exigiria manutenção prolongada de anestesia ou sedação dos animais de experimentação $0^{30}$. Transdutores cutâneos para análise da eletromiografia gástrica ainda estão em fase de avaliação; a grande dificuldade é filtrar através da parede abdominal aqueles impulsos que provêm do estômago e diferenciá-los de impulsos da musculatura da parede abdominal ou de alças intestinais interpostas ${ }^{34}$.

Aceita-se, atualmente, a avaliação da atividade motora antroduodenal, realizada com equipamentos eletrônicos, como metodologia padrão para esse tipo de doença, ${ }^{3,6}$. As sondas eletrônicas têm a vantagem de não necessitarem de sistema de perfusão líquida ou gasosa, condições essas que podem alterar determinados tipos de resultados. As sondas eletrônicas podem ser conectadas a sistemas portáteis de registro de dados, o que permite que os cães se movimentem livremente no respectivo ambulante, diminuindo o estresse do procedimento, eacima de tudo, permitindo que $o$ procedimento de registro de dados seja realizado com o animal acordado, sem sedação. Como não encontraram-se dados sobre a manometria antroduodenal com cateteres eletrônicos em cães, foi necessário que as medições fossem realizadas no pré-operatório para a validação da técnica e elaboração de dados de referência. O período de validação do experimento demonstrou que na fase interdigestiva são encontrados os mesmos padrões de motilidade que pelo uso de manometria de perfusão, e esses, por sua vez, coincidem com os padrões de eletromiografia correspondentes.

Com a ressecção da área correspondente ao marca-passo gástrico, foi possível identificar diversas alteraçõ̃es no padrão de motilidade antroduodenal, porém essas não foram do tipo redução no tempo de transporte do quimo. Tanto no antro gástrico quanto no duodeno, observou-se IMC's completos após a ressecção do marca-passo gástrico. Disso pode-se concluir que os centros de estimulação secundários têm a capacidade de gerarem os padrões típicos de motilidade 9 . O presente estudo não teve por objetivo estudar se a redução volumétrica do fundo gástrico altera de alguma forma a qualidade do quimo, nem monitorar o tempo de esvaziamento gástrico.

A manometria de antro gástrico realizada no pré-operatório demonstrou, na média geral das fases, participação maior da fase 2, seguida da fase 3 e da fase I. No pós-operatório, observou-se no estômago diminuição na participação das fases I e 3 e aumento na participação percentual de ondas típicas de fase 2; a diminuição da fase I (a fase de inatividade do estômago) não foi observada por Marike Code, que realizaram vagotomia, demonstrando assim a independência dos dois mecanismos de regulação, ou seja, a ressecção do marca-passo gástrico não tem o mesmo efeito sobre a fase I do que a vagotomia ${ }^{15}$. Jáa diminuição da fase 3 , observada no presente estudo, também foi observada por Gleysteen, Sarna e Myrvik após vagotomia reversíve ${ }^{20} .0$ comportamento das fases foi semelhante no duodeno, com aumento da fase 2 e diminuição da fase 3, demonstrando a íntima inter-relação funcional entre ambas, fato reforçado pela concordância de resultados para os parâmetros de número e duração dos IMC's no préoperatório entre estômago e duodeno. Nãoé possível concluir, com os dados elaborados, se a diminuição observada na participação da fase I e o aumento na participação da fase 2 no IMC do antro gástrico interferem sobre o refluxo duodenogástrico. Mearin, Azpiroz, Malagelada et al. ressaltam que durante a fase I do estômago de cães, o tônus pilórico é menor, aumentando gradativamente nas fases 2 e 3, e que o refluxo duodenogástrico é menor na fase I, com maior pico na fase 2 . Todavia, o piloro não é o elemento principal na prevenção do refluxo duodenogástrico. Observou-se, na presente série de cães, redução na linha de base do $\mathrm{pH}$ gástrico devido ao aumento no número de IMC's no duodeno, 0 que, por sua vez, demonstra que houve menor participação de episódios de refluxo duodenogástrico. Isso significa que o aumento na fase 2 do antro, por si só, não determina a ocorrência de refluxo duodenogástrico, porém este ocorre provavelmente como resulta- 
do da interação de diversos fatores ${ }^{18}$.

No presente estudo, observou-se para o antro gástrico de cães aumento no índice de motilidade na fase 3 , o que se explica pelo aumento significativo da amplitude das ondas, concomitante à diminuição da duração do tempo da fase 3. Não é possível determinar, com os dados atuais, se esse aumento no índice de motilidade é devido à redução do volume de acomodação do estômago ou devido à retirada da área correspondente ao marca-passo gástrico, ou ainda devido à interação de ambos os fatores" ". Para criar tal protocolo, a dificuldade seria mapear no intra-operatório - local exato do marca-passo na curvatura gástrica maior, eaír ressecá-lo sem comprometimento do fundo gástrico; mesmo que isso fosse possível de ser realizado, haveria dificuldades de interpretação dos resultados quanto à influência da retração cicatricial no estômago proximal sobre a capacidade de recepção e relaxamento do mesmo ${ }^{17,35}$.

Tendências divergentes entre antro gástrico e duodeno foram observadas para a freqüência durante o período pós-prandial, a qual foi maior no antro gástrico em relação à avaliação total; no duodeno observou-se tendência contrária, de aumento da freqüência. Provavelmente, as alterações funcionais observadas na fase interdigestiva estão relacionadas à ressecção do marca-passo gástrico.

Os fatores que participam na determinação do pH no compartimento gástrico são as secreções ácida e mucosa, ingestão alimentar, deglutição de saliva, refluxo de secreção biliar, duodenal e pancreática e ainda a eficiência na mistura e transporte do quimo para o duodeno. Utilizaram-se os critérios de Fuchs, DeMeester, Hinder et al., os quais ressaltam que para que se possa detectar anormalidades de $\mathrm{pH}$, considerando-se os vários fatores determinantes do $\mathrm{pH}$, deve-se fazer análise da freqüência de distribuição do pH; para tanto, pode-se dividi-lo em intervalos de $\mathrm{pH}$, por exemplo, I-2, 2-3, etc. Assim, pode-se avaliar a percentagem de tempo ou freqüência que a luz gástrica ficou exposta a cada intervalo de $\mathrm{pH}^{2}$. Observou-se deslocamento da linha de base para mais ácida, tendência também observada nos diversos intervalos de $\mathrm{pH}$ mensurados, que demonstram aumento nos intervalos mais ácidos. Pode-se interpretar essa queda no $\mathrm{pH}$ gástrico pela observação de maior número de IMC's no duodeno, o que poderia explicar o menor número de episódios de refluxo duodenogástrico. Resultados semelhantes foram descritos em estudos que avaliaram a motilidade duodenal após procedimentos de gastrectomia ${ }^{33}$. Fuchs, DeMeester, Hinder et al. encontraram linha de base do $\mathrm{pH}$ canino com variação entre $\mathrm{pH}$ I e 4, ressaltando maior acidez nas porções mais proximais do estômago ${ }^{2}$. O aumento percentual da fase 2 na atividade motora do antro gástrico não contribuiu para a alcalinização do estômago, como seria de se esperar, devido às alterações concomitantes do IMC observadas no duodeno.

O presente estudo abre diversas perspectivas de pesquisa na área da motilidade antroduodenal. Os dados elaborados sugerem que ocorre a participação de múltiplos fatores na determinação da motilidade no período interdigestivo do estômago. A relação entre 0 aumento da acidez gástrica e da freqüência de IMC's no duodeno deverá ser estudada pela identificação de bile no estômago, correlacionando-a com os IMC's duodenais.

Com base nos resultados elaborados, demonstrou-se a aplicabilidade das sondas de manometria eletrônica e de $\mathrm{pH}$-metria no modelo canino. Pode-se concluir que pela retirada da área do provável marca-passo gástrico ocorreram alterações de motilidade antroduodenal, permanecendo porém a ocorrência de IMC's completos; isso sugere que regiões subordinadas passaram a assumir a função de marca-passo. Quanto ao $\mathrm{pH}$, houve redução do tempo prandial e acidificação da linha de base gástrica.

\section{SUMMARY}

Antroduodenal MOtILITY AND PH CHANGES AFTER PARTIAL GASTRECTOMY INCLUDING THE PACEMAKER REGION OF THE DOG'S STOMACH

BACKGROUND. Functional alterations of antroduodenal motility are not well defined at present. The gastric pacemaker is located at the greater curvature, next to the fundus.

PURPOSE. The aim was to evaluate the influence of the gastric pacemaker on the gastroduodenalmotility in dogs.

METHODS. The study was designed in three steps and performed with 20 dogs: first, construction of a cervical esophagostomy to measure the antroduodenal motility and gastric $\mathrm{pH}$-metryas validation step. The manometry was performed by means of a six pointelectronic manometryprobeand bothmanometryand $\mathrm{pH}$ metry were recordedduring 4 hours. Second, the stomach's pacemaker region and gastric reservoir were resected by videolaparoscopy. Third, the antroduodenal motility and gastric $\mathrm{pH}$-metryweremeasured again.

RESULTS. In the validation step, there was established a typical dogs' antroduodenal motility pattern, with a three-shaped interdigestive motility complex (IMC) (phases I to 3); the gastric $\mathrm{pH}$-metry showed a baseline at $\mathrm{pH}$ I, I5. After partial gastric resection, overall there was an increase in contractions' frequency, an increase in phase 2 and a decrease in phase 3 participation on the IMC's. Regarding the gastric $\mathrm{pH}$ measurements, there was an increase in $\mathrm{pH}$ intervals $\mathrm{O}-\mathrm{I}$ and $\mathrm{I}-2$ and a decrease in $\mathrm{pH}$ intervals 6-7; overall, there was a decrease in the stomach's $\mathrm{pH}$ and a decrease in the prandial time.

CONCLUSION. The resection of thestomach's pacemaker region and gastric reservoir correlated with alterations in antroduodenal motility, maintaining however the typical IMC pattern.[Rev Assoc Med Bras 2003; 49(2): 196-202]

KEY WORDS: Gastric pacemaker. Antroduodenal manometry. pH-metry. Videolaparoscopy. Dogs.

\section{RefERÊNCIAS}

I. QuigleyJP, Louckes HS. Gastric emptying. Am J Dig Dis 1962; 7:672-6.

2. Fuchs KH, DeMeester T, Hinder RA. Computerized identification of pathologic duodenogastric reflux using 24-hour gastric $\mathrm{pH}$ monitoring. Ann Surg 1990; 21 3:13-20.

3. Camilleri M. Study of human gastroduodenojejunal motility. Applied physiology in clinical practice. Dig Dis Sci 1 993; 38:785-94.

4. Wingate DL, Stacher G, Kreiss C. Role and integration of mechanisms controling gastric emptying. Dig Dis Sci 1 994; 39: I 20S-3S.

5. Fuchs KH, Dietz UA, Heimbucher J, Fein M, Malafaia $\mathrm{O}$, Thiede $\mathrm{A}$. The role of gastric functional disorders in gastroesophageal reflux disease. ABCD Arq Bras Cir Dig 1996; I I:336.

6. Byrne KG, Quigley EM. Antroduodenal manonetry: an evaluation of an emerging methodology. Dig Dis 1997; I5(Suppl I):53-63.

7. Malagelada JR, Stanghellini V. Manometric evaluation of functional upper gut symptoms. 
Gastroenterology 1985; 88: | 223-31.

8. Verhagen MA, Samsom M, Smout AJ. Gastric myoelectrical and antroduodenal motor activity in patients with achalasia. Neurogastroenterol Motil 1998; 10:21 I-8.

9. Weber J, Kohatsu S. Pacemaker localization and electrical conduction patterns in the canine stomach. Gastroenterology 1 970; 59:7। 7-26.

10. Chiba T, Ohi R, Kamiyama T, Yoshida S, Hongo $M$. Does the stomach remain silent after neonatal loss of its original pacemaker?: gastric motility in long-term survivors of neonatal gastric rupture. Tohoku J Exp Med 1999; 187:89-97.

II. Cannon WB, Lieb CM. The receptive relaxation of the stomach. Am J Physiol I9II; 29:270-3.

12. Stadaas JO. Intragastric pressure/volume relationsship before and after proximal gastric vagotomy. Scand J Gastroenterol 1975; 10:129-34.

13. SzurszewskiJH. A migrating electric complex of the canine small intestine. Am J Physiol I 969; 217:1757-63.

14. Ehrlein JH, Bühner S, Thoma G. Gastric emptying after Roux-Y and Billroth I gastrectomy depends on viscosity of meal and contractile patterns of small intestine in dogs. Dig Dis Sci 1987; 32:529-37.

15. Marik F, Code $\mathrm{CH}$. Control of the interdigestive myoeletric activity in dogs by the vagus nerves and pentagastrin. Gastroenterology 1975; 69:387-95.

16. Sarna SK. Cyclic motor activity; migrating motor complex: 1985. Gastroenterology 1985; 89:894-9|3.

17. Dalton RR, Zinsmeister AR, Sarr MG. Vagusdependent disruption of interdigestive canine motility by gastric distension. Am J Physiol 1992; 262:1097-103.
18. Mearin F, Azpiroz F, MalageladaJR, Zinsmuster AR. Antroduodenal resistance to flow in the control of duodenogastric bile reflux during fasting. Gastroenterology 1987; 93: 1026-33.

19. Duthie HL, Brown BH, Robertson-Dunn B, Kwong NK, Whittaker GE, Waterfall W. Electrical activity in the gastroduodenal area slow waves in the proximal duodenum. A comparison of man and dog. Dig Dis Sci 1972; 17:344-5|.

20. Gleysteen JJ, Sarna SK, Myrvik AL. Canine cyclic motor activity of stomach and small bowel: the vagus is not the governor. Gastroenterology 1984; 88: 1926-31.

2I. Sarna S, Stoddard C, Belbeck L, Mc Wade D. Intrinsic nervous control of migrating myoelectric complexes. Am J Physiol 1981; 24I:GI6-23.

22. Kelly KA, Code CF. Effect of transthoracic vagotomy on canine gastric electric activity. Gastroenterology 1969;57:51-8.

23. Hinder RA, Bremner CG. Relative role of pyloroplasty size, truncal vagotomy and milk meals volume in canine gastric emptying. Am J Dig Dis 1978; 23:210-6.

24. DiMarino A, Carlson G, Myers A, Schumacher $\mathrm{HR}$, Cohen S. Duodenal myoelectric activity in scleroderma. NEngl J Med 1973; 289: 1220-3.

25. Fealy RD, SzurszewskiJH, Merritt JL, Di Magno EP. Effect of spinal cord transsection on human gastrointestinal motility and gastric emptying. Gastroenterology 1984; 87:69-75.

26. Fich A, Neri M, Camilleri M, Kelly KA, Phillips SF. Stasis syndromes following gastric surgery: clinical and motility features of 60 symptomatic patients. J Clin Gastroenterol 1990; 12:505-12.

27. Summers GE, Hocking MP. Preoperative and postoperative motility disorders of the stomach. Surg Clin North Am 1 992; 72:467-86.
28. Wingate DL, Ruppin H, Green WER, Thompson HH, DomschkeW, Wunsch E etal. Motilin-induced eletrical activity in the canine gastrointestinal tract. Scand J Gastroenterol 1976; II: III-5.

29. Sonnenberg A, Müller-Lissner SA, Schattenmann G, Siewert JR, Blum AL. Duodenogastric reflux in the dog. Am J Physiol 1982; 242:G603-G7.

30. Valori RM, Collins SM, Daniel EE, Reddy SN, Shannon S, Jury J. Comparison of methodologies for the measurement of antroduodenal motor activity in the dog. Gastroenterology 1986; 91:546-53.

31. Stevens RJ, Weinert JS, Publicover NG. Visualization of origins and propagation of excitation in canine gastric smooth muscle. Am JPhysiol 1999; 277:C448-C60.

32. Dent J. A new technique for continuous sphincter pressure measurement. Gastroenterology 1976; 71:263-7.

33. Heimbucher J, Fuchs KH, Freys SM, Clark GW, Incarbone R, De Meester TR et al. Motility in the Hunt-Lawrence pouch after total gastrectomy. Am J Surg 1994; 168:622-6.

34. Geldorf $H$, Van der Schede EJ, Van Blankenstein M, Smout AJ, Akkermans LM. Effects of highly selective vagotomy on gastric myoelectrical activity. An electrogastrografic study. Dig Dis Sci 1990; 35:969-75.

35. Kelly KA. Gastric motility after gastric operations. Surg Annu 1974; 6: 103-23.

Artigo recebido: 21/03/2002 Aceito para publicação: I I/09/2002 